\title{
A successful management of acute cholemic nephropathy secondary to acute liver abscess and pylephlebitis associated severe sepsis by ERCP, combined CVVHDF and stepwise hemodialysis
}

\begin{abstract}
Cholemic nephrosis or yellow kidneys, is a rare condition and can be associated with acute kidney injury secondary to various etiologies. We are reporting a difficult case presented with fatal severe sepsis associated with hepatic, pancreatic and renal injury secondary to impacted biliary stone at the ampulla of vater and complicated by infection. With proper management and early drainage of biliary system and continuous renal replacement therapy the patient survived. The early recognition and interference leads to reversal of symptoms and improvement of renal and patient survival.
\end{abstract}

Keywords: acute hepatitis, cholestatic hepatitis, septic shock, acute kidney injury, renal replacement therapy, ascending cholangitis
Volume 9 Issue 2 - 202।

\author{
Nour R Al-Khatib,' Ali Awil Al, ${ }^{4}$ Renad Al- \\ Shobaki,' Salem M Bazarah,' Nabih Hamad,' \\ Wail Tushkandi, ${ }^{2}$ Ahmed Akl ${ }^{1,3,5}$ \\ IInternal Medicine Department, Dr Soliman Fakeeh Hospital, \\ Jeddah, KSA \\ IIntensive Care Department, Dr Soliman Fakeeh Hospital, \\ Jeddah, KSA \\ ${ }^{3}$ Fakeeh College of medical sciences, Jeddah, KSA \\ ${ }^{4}$ Internal Medicine Department, King Fahad Hospital, Jeddah, \\ KSA \\ 5Urology \& Nephrology center, Mansoura University, Mansoura, \\ Egypt
}

Correspondence: Ahmed AkI MD, FACP, FASN, DR. Soliman Fakeeh hospital, Jeddah, KSA, Urology \& Nephrology center Mansoura University, Egypt, Email aiak1200।@yahoo.com

Received: March 21, 202I | Published: April 16, 2021

\section{Introduction}

Cholestatic liver disease affecting kidney function by different mechanisms and is described by different names: bile cast nephropathy or cholemic nephrosis, biliary nephrosis, jaundicerelated nephropathy or yellow kidneys. ${ }^{1}$ It carry a various hazards on kidney function ranging from hemodynamic changes accounting for pre-renal hypo-perfusion, ischemic injury and direct tubular toxicity induced by hyperbilirubinemia and bile casts. ${ }^{1,2}$ Its prevalence is increasing with more reporting of identified cases compared to previously documented. ${ }^{3-8}$ The early recognition and interference leads to reversal of symptoms and improvement of renal and patient survival. Choledocholithiasis, is associated with acute kidney injury is accompanied by typical histological changes, including tubular epithelial injury, together with intraluminal bile cast formation., ${ }^{9,10}$

Biliary tract disease is the most common condition associated with liver abscess. ${ }^{11}$ This includes infection (cholangitis) that may occur secondary to choledocholithiasis, biliary stricture, or malignancy. ${ }^{12}$

\section{Case report}

A 59-year-old female with multiple co-morbidities including ischemic heart disease with coronary artery bypass grafting in 2016, diabetes mellitus, hypertension. She presented with fever, abdominal pain related to chronic calcular cholecystitis and poor oral intake referred to examined by general surgery and they recommend to start antibiotics course and to be re-assessed again in a week time. After four days, patient came to the emergency department with severe septic shock, blood pressure as low as $80 / 40 \mathrm{mmHg}$ refractory to fluids resuscitation. Radiological investigations revealed severe ascending cholangitis, impacted biliary stone at level of ampulla of vater, complicated by liver abscess and anuric acute kidney injury with refractory hyperkalemia and metabolic acidosis requiring urgent renal replacement therapy. Patient was transferred immediately to intensive care unit. In view of dilated biliary radicles with signs of biliary stone impaction, urgent endoscopic retrograde cholangiopancreatography (ERCP) was performed, with stent insertion and pulling out of a large pigmented stone and drainage of biliary muddy fluid (Figure 1). Immediate continuous renal replacement therapy in the form of continuous venovenous hemodiafiltration (CVVHDF) was initiated to correct the metabolic and electrolytes disturbances in order to save the patient life.

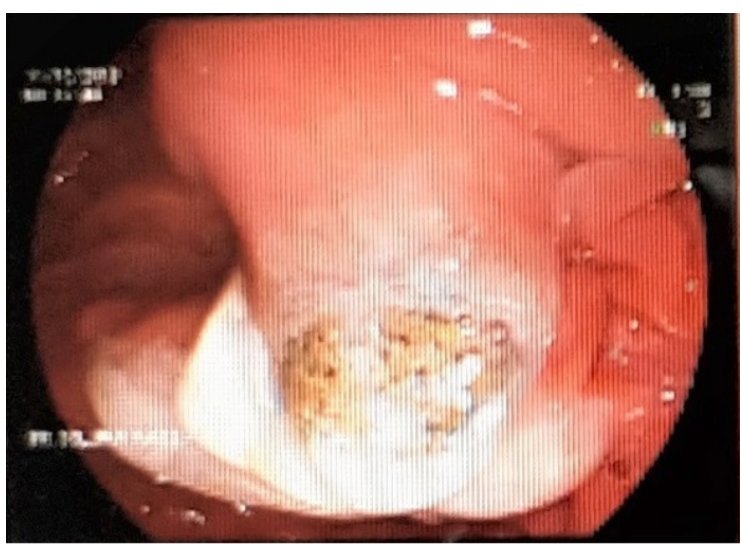

A) Pigmented biliary stone obstructing Ampula of Vater. 


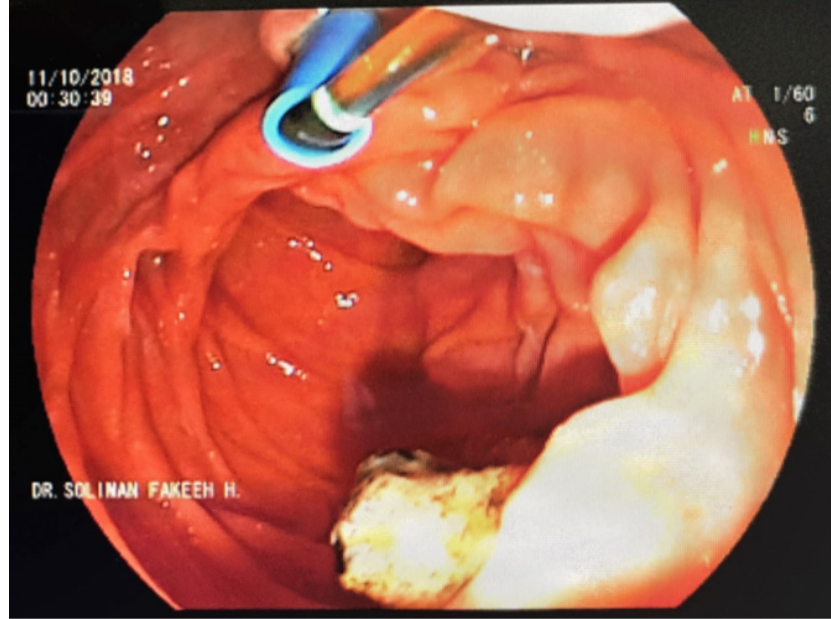

B) Stent of the common bile duct for drainage.

Figure I ERCP and dislodge of impacted biliary stone at ampulla of Vater.

Abdominal ultrasound imaging revealed a constant interval of the liver abscess and postal vein thrombosis (pylephlebitis). Pylephlebitis and severe cholangitis were managed by a total of 4 weeks of intravenous antibiotics and therapeutic heparin infusion. Aspiration of the liver abscess was done and culture revealed negative growth. Repeated abdominal us showed improved pylephlebitis and patent portal vein. The patient was evaluated by general surgery, with their recommendation being to postpone the cholecystectomy till after resolution of the liver abscess. She was discharged home after 4 weeks to complete 6 weeks to 3 months of antibiotics or until complete resolution of the abscess at outpatient follow up basis. Regarding the state of anuria in setting of acute kidney injury, the patient had required daily continuous renal replacement therapy in the intensive care unit for 1 week. Thereafter, her hemodynamic improved and shifted to slow inefficient dialysis daily (SLEDD). Clinically improved with improvement of her urine output (Figure 2) the need for hemodialysis declined to 3 times per week, then twice per week, till reaching a requirement of once per week. The patient's urine output improved to around $2 \mathrm{~L} /$ day in the few days prior to discharge, with her last creatinine clearance estimated to be $10 \mathrm{ml} / \mathrm{min}$. She was discharged on furosemide twice per oral daily and bicarbonate tablets, as well as oral anti-hypertensive medications. At the outpatient clinic, her kidney function improved with serum creatinine reaching basal level $1.8 \mathrm{mg} / \mathrm{dl}$, eGFR (EPI) $30 \mathrm{ml} / \mathrm{min}$ and normal electrolytes with no hemodialysis. Her abdominal us shows normal biliary radicles and patent portal vein.

\section{FLUID CHART}

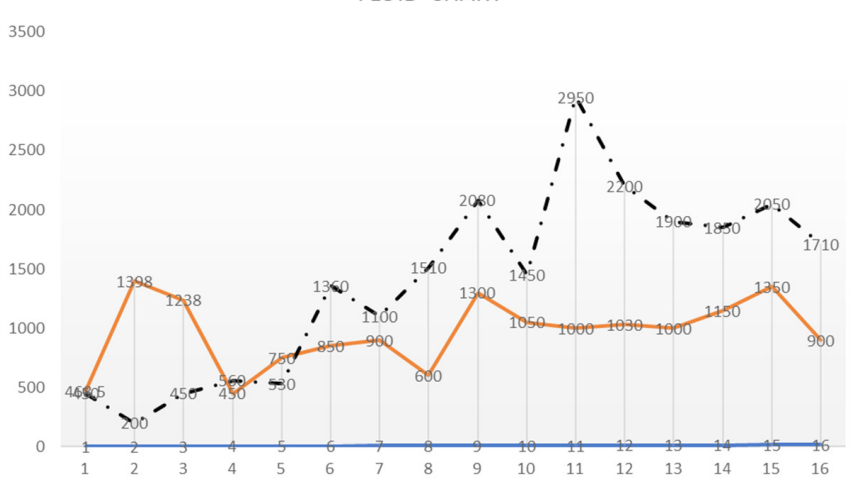

Figure 2 Daily monitoring of fluids intake and urine output.

\section{Discussion}

The mechanisms of kidney injury described with bile cast nephropathy is similar to to the injury caused by myeloma or myoglobin casts. Bile casts may lead to oxidative stress and trigger the inflammasome signaling cascade, leading to interstitial inflammation. ${ }^{13}$ Definitive diagnosis of bile cast nephropathy is by renal biopsy, as there is no validated non-invasive specific diagnostic test and it is frequently diagnosed at time of autopsy or on kidney biopsy. ${ }^{13}$ Histological land marks appears in distal tubules in the form of dilated tubules and obstruction by intraluminal casts. The bile casts can be confirmed by periodic-acid Schiff (PAS) stains showing red to dark red colored casts ${ }^{15}$ or Masson trichrome using aniline green, bile casts show a green color. ${ }^{16}$ Besides bile casts, kidney histology may show varying degrees of acute tubular injury characterized by tubular epithelial cells with attenuated cytoplasm or loss of proximal tubular brush borders or regenerative changes and interstitial inflammation. ${ }^{15}$

Also, the presence of mononuclear inflammatory cells in the vasa recta has been previously reported ${ }^{15}$ Given the current lack of noninvasive methods to diagnose bile cast nephropathy and that kidney histology is the single established diagnostic test, conventional kidney biopsy is relatively risky in patients with liver disease, especially with impaired blood coagulation. Additionally, distal nephron segments, which have been reported to be of specific diagnostic importance for establishing the diagnosis of $\mathrm{CN}$, are probably not reached by conventional kidney biopsy, since this also carries a significant risk for bleeding. Whether a trans-jugular approach may represent a suitable and safe alternative to significantly reduce such risks in this group of patients as was shown. ${ }^{17}$ In our case we could not do renal biopsy because of the severe sepsis and coagulopathy status. Due to the rarity of bile cast nephropathy, there are currently no established treatment guidelines. Interventions to reduce bilirubin burden, such as relief of biliary obstruction via ERCP with stent placement and hemodialysis, have been attempted to improve outcomes. While this appears to be an effective strategy early in the disease course, its efficacy in established disease is uncertain. ${ }^{18-21}$ Plasmapheresis may additionally be beneficial. ${ }^{22}$

\section{Conclusion}

In conclusion, early diagnosis of obstructive cholangitis and drainage which followed by hemodiafiltration improved patient condition and enhanced recovery from severe sepsis, hyperbilirubinemia and bile salt nephropathy.

\section{Acknowledgments}

We would like to thank our ICU, RDU, Inpatient nurses teams, our residents and all other hospital stuff for their hard work taking care of our patient.

\section{Conflicts of interest}

The author declares there is no conflict of interest.

\section{References}

1. van Slambrouck CM, Salem F, Meehan SM, et al. Bile cast nephropathy is a common pathologic finding for kidney injury associated with severe liver dysfunction. Kidney Int. 2013;84(1):192-197.

2. Sitprija V, Kashemsant U, Sriratanaban A, et al. Renal function in obstructive jaundice in man: cholangiocarcinoma model. Kidney Int. 1990;38(5):948-955.

3. Bredewold OW, de Fijter JW, Rabelink T. A case of mononucleosis infectiosa presenting with cholemic nephrosis. NDT Plus. 2011;4(3):170 172.

Citation: Al-Khatib NR, Al AA, Al-Shobaki R, et al. A successful management of acute cholemic nephropathy secondary to acute liver abscess and pylephlebitis associated severe sepsis by ERCP, combined CVVHDF and stepwise hemodialysis. Urol Nephrol Open Access J. 202I;9(2):43-45. DOI: I0.15406/ unoaj.2021.09.00306 
4. Betjes MG, Bajema I. The pathology of jaundice-related renal insufficiency: cholemic nephrosis revisited. J Nephrol. 2006;19(2):229 233.

5. Luciano RL, Castano E, Moeckel G, et al. Bile acid nephropathy in a bodybuilder abusing an anabolic androgenic steroid. Am J Kidney Dis. 2014;64(3):473-476.

6. van der Wijngaart H, van Dam B, van den Berg JG, et al. A 73-year-old male with jaundice and acute kidney injury. Neth J Med. 2014;72:95-99.

7. Sequeira A, Gu X. Bile cast nephropathy: an often forgotten diagnosis. Hemodial Int. 2015;19:132-135.

8. Jain K, Gupta A, Singh HK, et al. Bile cast nephropathy. Kidney Int 2015;87:484

9. Krones E, Wagner M, Eller K, et al. Bile acid-induced cholemic nephropathy. Dig Dis. 2015;33:367-375.

10. Uslu A, Tas, li FA, Nart A, et al. Human kidney histopathology in acute obstructive jaundice: a prospective study. Eur J Gastroenterol Hepatol. 2010;22:1458-1465.

11. Alvarez Perez JA, Gonzalez JJ, Baldonedo RF, et al. Clinical course, treatment, and multivariate analysis of risk factors for pyogenic liver abscess. Am J Surg. 2001;181(2):177-186.

12. Wong TY, Chiu SI, So MK, et al. Septic metastatic endophthalmitis complicating Klebsiella pneumoniae liver abscessin a non-diabetic Chinese man. Hong Kong Med J. 2001;7(3):303-306.

13. Kurt T K Giuliani, Andrew J Kassianos, Katrina Kildey, et al. Role of inflammation and inflammasome activation in human bile cast nephropathy. Nephrology (Carlton). 2020;5(6):502-506
14. Elisabeth Kronesa, Marion J Pollheimer, Alexander R Rosenkranz, et al. Cholemic nephropathy - Historical notes and novel perspectives. Biochimica et Biophysica Acta (BBA) - Molecular Basis of Disease. 2018;1864(4):1356-1366.

15. van Slambrouck CM, Salem F, Meehan SM, et al. Bile cast nephropathy is a common pathologic finding for kidney injury associated with severe liver dysfunction. Kidney Int. 2013;84(1):192-197.

16. Julien Aniort, Anaïs Poyer, Jean-Louis Kemeny, et al. Bile Cast Nephropathy Caused by Obstructive Cholestasis. Kidney Biopsy Teaching Case. 2016;69(1):143-146.

17. JM Trawale, V Paradis, PE Rautou, et al. The spectrum of renal lesions in patients with cirrhosis: a clinicopathological study. Liver Int off $J$ Int Assoc Study Liver. 2010;30(5):725-732.

18. Betjes MG, Bajema I. The pathology of jaundice-related renal insufficiency: cholemic nephrosis revisited. J Nephrol. 2006;19:229-233.

19. van der Wijngaart H, van Dam B, van den Berg JG, et al. A 73-year-old male with jaundice and acute kidney injury. Bile cast nephropathy. Neth $J$ Med. 2014;72(2):95-99.

20. Sequeira A, Gu X. Bile cast nephropathy: an often forgotten diagnosis. Hemodial Int. 2015;19:132-135.

21. Speer AG, Cotton PB, Russell RC, et al. Randomised trial of endoscopic versus percutaneous stent insertion in malignant obstructive jaundice. Lancet. 1987;2(8550):57-62.

22. Ocon AJ, Rosenblum M, Desemone J, et al. Severe cholestatic hyperbilirubinaemia secondary to thyrotoxicosis complicated with bile cast nephropathy treated with plasma exchange and haemodialysis. $B M J$ Case Reports CP. 2019;12(6):e229097. 